\title{
A New Approach to Global Stability of Discrete Lotka-Volterra Predator-Prey Models
}

\author{
Young-Hee Kim ${ }^{1}$ and Sangmok $\mathrm{Choo}^{2}$ \\ ${ }^{1}$ Division of General Education-Mathematics, Kwangwoon University, Seoul 139-701, Republic of Korea \\ ${ }^{2}$ Department of Mathematics, University of Ulsan, Ulsan 680-749, Republic of Korea \\ Correspondence should be addressed to Sangmok Choo; smchoo@ulsan.ac.kr
}

Received 24 March 2015; Accepted 20 May 2015

Academic Editor: Ryusuke Kon

Copyright (c) 2015 Y.-H. Kim and S. Choo. This is an open access article distributed under the Creative Commons Attribution License, which permits unrestricted use, distribution, and reproduction in any medium, provided the original work is properly cited.

An Euler difference scheme for a three-dimensional predator-prey model is considered and we introduce a new approach to show the global stability of the scheme. For this purpose, we partition the three-dimensional space and calculate the sign of the rate change of population of species in each partitioned region. Our method is independent of dimension and then can be applicable to other dimensional discrete models. Numerical examples are presented to verify the results in this paper.

\section{Introduction}

Vito Volterra proposed a differential equation model to explain the observed increase in predator fish and corresponding decrease in prey fish in 1926. At the same time, the equations studied by Volterra were derived independently by Alfred Lotka (1925) to describe a chemical reaction. Many predator-prey models have been studied and a classic predator-prey model is given by

$$
\begin{aligned}
& \frac{d x}{d t}=x\left(r_{1}-a_{11} x-a_{12} y\right), \\
& \frac{d y}{d t}=y\left(-r_{2}+a_{21} x-a_{22} y\right),
\end{aligned}
$$

where $r_{i}>0, a_{i j}>0$, and $x$ and $y$ denote the population sizes of the prey and predator, respectively.

It is recognized that the rate of prey capture per predator cannot increase indefinitely as the number of prey increases. Instead, the rate of prey capture is saturated when the population of prey is relatively large. Then such nonlinear functional responses are employed to describe the phenomena of predation including the Holling types [1-5], BeddingtonDeAngelis type [6-8], Crowley-Martin type [9-11], and Ivlev type of functional response [12-14].
In particular, similar phenomena are observed in the interactions in chemical reactions and molecular events when one species is abundant. Thus linear response function, Michaelis-Menten kinetics, and Hill function are related to Holling types I, II, and III, respectively. Holling type IV is also called the Monod-Haldane function $[4,5]$.

The functional responses have been applied to predatorprey models to express the Allee effect [15-19], which describes a positive relation between population density and the per capita growth rate.

Most of researches on the predator-prey models assume that the distribution of the predators and prey is homogeneous, which leads to ordinary differential equations. However both predators and prey have the natural tendency to diffuse, so that there have been models to take into account the inhomogeneous distribution of the predators and prey [20-22].

On the other hand, population is inevitably affected by environmental noise in nature. Therefore, many authors have taken stochastic perturbation into deterministic models [2325].

There are a number of works investigating continuous time predator-prey models, but relatively few theoretical papers are published on their discretized models [26, 27]. As far as we know, there is no theoretical research on the 
global stability of the discrete-time models of type (1) with more than two species except for [28]. The author in [28] introduced a method to present global stability for the case that all species coexist at a unique equilibrium. Then a new approach needs to be developed for the other cases.

For explaining our new approach, we consider a model with one prey and two predators:

$$
\frac{d x^{i}}{d t}=x^{i}\left(\sigma_{i} r_{i}+\sum_{1 \leq j \leq i-1} a_{i j} x^{j}-\sum_{i \leq j \leq 3} a_{i j} x^{j}\right),
$$

where $i=1,2,3, \sigma_{1}=1, \sigma_{2}=\sigma_{3}=-1$, and

$$
\begin{aligned}
& \frac{r_{3}}{a_{31}}<\frac{r_{1}}{a_{11}}<\frac{r_{2}}{a_{21}}, \\
& \frac{r_{1}}{a_{12}}<\frac{r_{3}}{a_{32}}, \\
& \frac{a_{21}}{a_{23}}<\frac{a_{31}}{a_{33}} .
\end{aligned}
$$

Here $x^{1}$ denotes the population number of the prey; $x^{2}$ and $x^{3}$ denote the population numbers of the predators. Letting

$$
f_{i}\left(x^{1}, x^{2}, x^{3}\right)=\sigma_{i} r_{i}+\sum_{1 \leq j \leq i-1} a_{i j} x^{j}-\sum_{i \leq j \leq 3} a_{i j} x^{j},
$$

the Euler difference scheme for (2) is as follows:

$$
\frac{\Delta x_{k}^{i}}{\Delta t}=x_{k}^{i} f_{i}\left(x_{k}^{1}, x_{k}^{2}, x_{k}^{3}\right), \quad k=0,1, \ldots,
$$

where $x_{0}^{i}>0(i=1,2,3), \Delta x_{k}^{i}=x_{k+1}^{i}-x_{k}^{i}$, and $\Delta t$ is a step size.

The three conditions (3)-(5) mean that the two planes $f_{1}(x, y, z)=0$ and $f_{3}(x, y, z)=0$ intersect in the first octant, and the plane $f_{2}(x, y, z)=0$ is not intersected with either $f_{1}(x, y, z)=0$ or $f_{3}(x, y, z)=0$ in the first octant. For example, let $\left(r_{i}, a_{i 1}, a_{i 2}, a_{i 3}\right)(1 \leq i \leq 3)$ be

$$
\begin{aligned}
& (2,1,2,2), \\
& (3,1,1,1), \\
& (2,2,1,1),
\end{aligned}
$$

respectively. Then the three conditions (3)-(5) are satisfied and Figure 1 shows the three planes and regions with the globally stable point of scheme (7). The global stability of the point will be shown in Section 3.

Using Theorem 4.1 in [28], we have the positivity and boundedness of the solutions of scheme (7). Letting $\chi_{i}(1 \leq$ $i \leq 3$ ) satisfy

$$
\begin{aligned}
& \chi_{i}<\frac{1+\sigma_{i} r_{i} \Delta t-\sum_{i+1 \leq j \leq n} a_{i j} \chi_{j} \Delta t}{2 a_{i i} \Delta t}, \\
& \frac{\sigma_{i} r_{i}+\sum_{1 \leq j \leq i-1} a_{i j} \chi_{j}}{a_{i i}}<\chi_{i}, \\
& \left(\sigma_{i} r_{i}+\sum_{1 \leq j \leq i-1} a_{i j} \chi_{j}\right) \Delta t<1,
\end{aligned}
$$

we have that for all $k$

$$
\left(x_{k}^{1}, x_{k}^{2}, x_{k}^{3}\right) \in\left(0, \chi_{1}\right) \times\left(0, \chi_{2}\right) \times\left(0, \chi_{3}\right) .
$$

For small values of $\Delta t$, there exist infinitely many $\chi_{i}(1 \leq i \leq$ 3) satisfying (9).

Consider the five regions

$$
\begin{aligned}
\mathrm{I}= & \left\{(x, y, z) \in \mathcal{S} \mid f_{1}(x, y, z) \geq 0, f_{2}(x, y, z)\right. \\
& \left.<0, f_{3}(x, y, z) \leq 0\right\}, \\
\mathrm{II} & =\left\{(x, y, z) \in \mathcal{S} \mid f_{1}(x, y, z) \geq 0, f_{2}(x, y, z)\right. \\
& \left.<0, f_{3}(x, y, z) \geq 0\right\}, \\
\mathrm{III} & =\left\{(x, y, z) \in \mathcal{S} \mid f_{1}(x, y, z) \leq 0, f_{2}(x, y, z)\right. \\
& \left.<0, f_{3}(x, y, z) \leq 0\right\}, \\
\mathrm{IV} & =\left\{(x, y, z) \in \mathcal{S} \mid f_{1}(x, y, z) \leq 0, f_{2}(x, y, z)\right. \\
& \left.\leq 0, f_{3}(x, y, z) \geq 0\right\}, \\
\mathrm{V} & =\left\{(x, y, z) \in \mathcal{S} \mid f_{1}(x, y, z)<0, f_{2}(x, y, z)\right. \\
& \left.\geq 0, f_{3}(x, y, z)>0\right\},
\end{aligned}
$$

where $\mathcal{S}=\left\{(x, y, z) \in \mathbb{R}^{3} \mid x>0, y>0, z>0\right\} \cap$ $\prod_{1 \leq i \leq 3}\left(0, \chi_{i}\right)$ (see Figure 1$)$.

For convenience we denote the set I by

$$
\begin{gathered}
\mathrm{I}(+,-,-) \\
\text { or }(+,-,-),
\end{gathered}
$$

and then

$$
P \in \mathrm{I}(+,-,-)
$$

$$
\text { iff } f_{1}(P) \geq 0, f_{2}(P)<0, f_{3}(P) \leq 0 .
$$

We adapt similar notations for the other regions II to V.

Note that region I has the property

$$
\sup \{x \mid(x, y, z) \in \mathrm{I}(+,-,-)\} \leq \frac{r_{1}}{a_{11}},
$$

since $f_{1}(x, y, z)=r_{1}-a_{11} x-a_{12} y-a_{13} z \geq 0$ for all $(x, y, z) \in$ $\mathrm{I}(+,-,-)$.

\section{Method}

In this section we present theorems that describe our approach. The theorems will be used to obtain the global stability of scheme (7) in the next section.

Assume that $\Delta t$ satisfies

$$
\begin{aligned}
& \Delta t\left\{\chi_{1}\left(a_{11}+a_{13} \frac{a_{21}}{a_{23}}\right)+\chi_{2} a_{22}+\chi_{3}\left(a_{33}+a_{32} \frac{a_{13}}{a_{12}}\right)\right\} \\
& <1 \\
& \frac{\Delta t}{1-a_{33} \chi_{3} \Delta t} a_{31} \chi_{1}^{2}<\frac{a_{31} r_{2}-a_{21} r_{3}}{a_{21}} .
\end{aligned}
$$



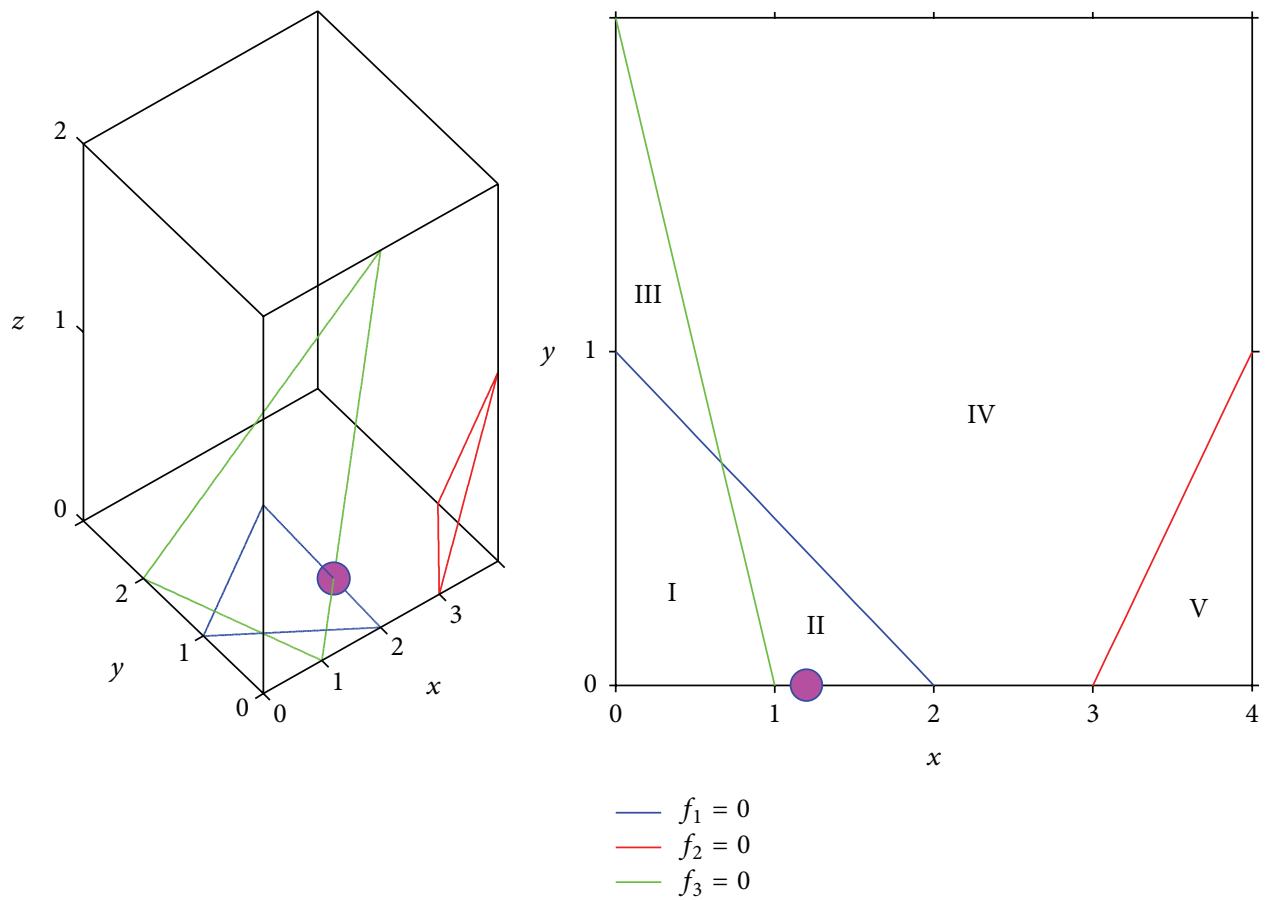

(a)

(b)

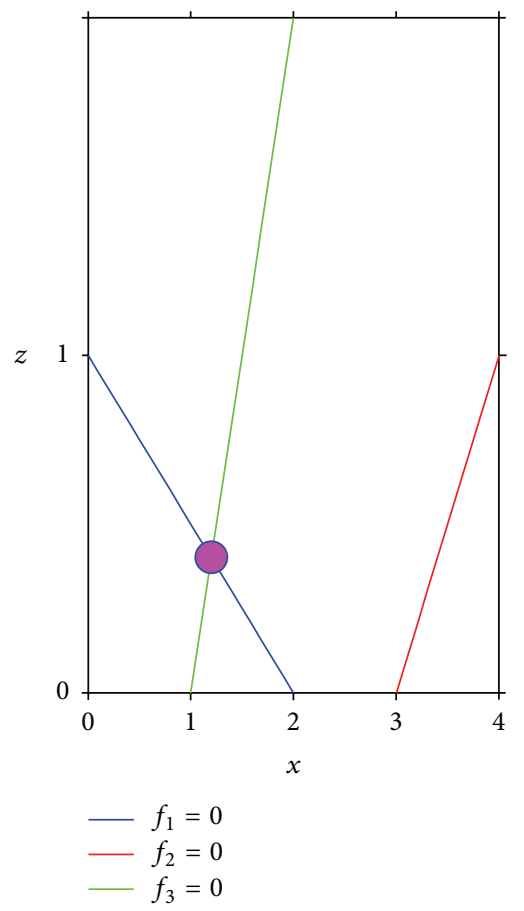

(c)

FIgURE 1: Three planes and regions with the globally stable point. (a) The three planes $f_{i}(x, y, z)=0(1 \leq i \leq 3)$ in $(0,4) \times(0,3) \times(0,2)$. The magenta circle denotes the globally stable point $(6 / 5,0,2 / 5)$ of scheme (7). (b) Five regions projected on the $x y$-plane. (c) Five regions projected on the $x z$-plane.

Let $P=\left(P_{1}, P_{2}, P_{3}\right)$ and $T=\left(T_{1}, T_{2}, T_{3}\right)$ be the vector function defined on $\mathcal{S}$ by

$$
T_{i}(P)=P_{i}\left\{1+\Delta t f_{i}(P)\right\}, \quad i=1,2,3 .
$$

Then scheme (7) can be written as

$$
\left(x_{k+1}^{1}, x_{k+1}^{2}, x_{k+1}^{3}\right)=T\left(x_{k}^{1}, x_{k}^{2}, x_{k}^{3}\right) .
$$


Note that the map $T$ has the three fixed points with all nonnegative components, $(0,0,0),\left(r_{1} / a_{11}, 0,0\right)$, and $\vartheta=$ $\left(\vartheta_{1}, 0, \vartheta_{3}\right)$, satisfying

$$
f_{1}(\vartheta)=f_{3}(\vartheta)=0 .
$$

Then the fixed point $\vartheta$ with $\vartheta_{1}=\left(r_{1} a_{33}+r_{3} a_{13}\right) /\left(a_{11} a_{33}+\right.$ $\left.a_{31} a_{13}\right)$ and $\vartheta_{3}=\left(r_{1} a_{31}-r_{3} a_{11}\right) /\left(a_{13} a_{31}+a_{33} a_{11}\right)$ is locally stable as follows.

Lemma 1. Let $\vartheta$ be the fixed point of the map $T$ satisfying (19). Assume that $\Delta t$ satisfies (15). Then the fixed point $\vartheta$ is asymptotically stable.

Proof. Applying the linearization method to the discrete system of (7) at $\vartheta$, the matrix of the linearized system has the three eigenvalues

$$
\begin{aligned}
1 & +f_{2}(\vartheta) \Delta t, \\
1 & -\frac{1}{2}\left(\vartheta_{1} a_{11}+\vartheta_{3} a_{33}\right) \Delta t \\
& \pm \frac{1}{2}\left\{\left(\vartheta_{1} a_{11}-\vartheta_{3} a_{33}\right)^{2}-4 \vartheta_{1} \vartheta_{3} a_{13} a_{31}\right\}^{1 / 2} \Delta t .
\end{aligned}
$$

Substituting $\vartheta_{1}=\left(r_{1} a_{33}+r_{3} a_{13}\right)\left(a_{11} a_{33}+a_{31} a_{13}\right)^{-1}$ to

$$
f_{2}(\vartheta)=-r_{2}+a_{21} \vartheta_{1}-a_{23} \vartheta_{3}<-r_{2}+a_{21} \vartheta_{1} \text {, }
$$

and using (3), we obtain

$$
f_{2}(\vartheta)<0 .
$$

In addition, (15) gives that the other two eigenvalues have magnitudes less than 1 . Hence the spectral radius of the matrix is less than 1 , which completes the proof.

The two fixed points $(0,0,0)$ and $\left(r_{1} / a_{11}, 0,0\right)$ are unstable since the matrices of the linearized system at $(0,0,0)$ and $\left(r_{1} / a_{11}, 0,0\right)$ have the eigenvalues $1+f_{1}(0,0,0) \Delta t$ and $1+$ $f_{3}\left(r_{1} / a_{11}, 0,0\right) \Delta t$ with $f_{1}(0,0,0)>0$ and $f_{3}\left(r_{1} / a_{11}, 0,0\right)>$ 0 , respectively. Then we only consider the fixed point $\vartheta=$ $\left(\vartheta_{1}, 0, \vartheta_{3}\right)$ of the map $T$ to show global stability in the next section.

Our methodology to obtain the global stability is based on the approach to determine regions among regions I to $\mathrm{V}$ in which $T(P)$ is contained by calculating the sign of $f_{i}(T(P))$ for $P$ in each region. Then we use the sign symbol $P \in(+, *, *)$ as follows.

$P \in(+, *, *)$ if and only if $f_{1}(P) \geq 0$ and the signs of $f_{i}(P)(i=2,3)$ are unknown.

Other sign symbols are similarly defined.

Theorem 2. Suppose that $\Delta t$ satisfies (9) and (15). Let $s \in$ $\{+,-\}$ :

If $P \in(s,-s,-s)$, then the sign of $f_{1}(T(P))$ is $s$.
Proof. Let $P=\left(P_{1}, P_{2}, P_{3}\right)$. Using the definition of $f_{1}$, we have

$$
\begin{aligned}
f_{1}(T(P))= & r_{1}-a_{11} T_{1}(P)-a_{12} T_{2}(P)-a_{13} T_{3}(P) \\
= & r_{1}-\sum_{1 \leq i \leq 3} a_{1 i} P_{i}\left\{1+\Delta t f_{i}(P)\right\} \\
= & r_{1}-\sum_{1 \leq i \leq 3} a_{1 i} P_{i}-\sum_{1 \leq i \leq 3} \Delta t a_{1 i} P_{i} f_{i}(P) \\
= & f_{1}\left(P_{i}\right)-\sum_{1 \leq i \leq 3} \Delta t a_{1 i} P_{i} f_{i}(P) \\
= & \left(1-\Delta t a_{11} P_{1}\right) f_{1}(P) \\
& -\Delta t\left\{a_{12} P_{2} f_{2}(P)+a_{13} P_{3} f_{3}(P)\right\}
\end{aligned}
$$

Note that if $f_{2}(x, y, z)=0$ for nonnegative $x, y$, and $z$, then

$$
x=\frac{1}{a_{21}}\left(r_{2}+a_{22} y+a_{23} z\right),
$$

with which the nonnegativity of $x, y$, and $z$ gives

$$
\begin{aligned}
f_{1}(x, y, z)= & r_{1}-a_{11} x-a_{12} y-a_{13} z \\
= & r_{1}-a_{11} \frac{1}{a_{21}}\left(r_{2}+a_{22} y+a_{23} z\right)-a_{12} y \\
& -a_{13} z \leq r_{1}-a_{11} \frac{1}{a_{21}} r_{2} \\
= & a_{11}\left(\frac{r_{1}}{a_{11}}-\frac{r_{2}}{a_{21}}\right)<0,
\end{aligned}
$$

and similarly

$$
f_{3}(x, y, z)>0,
$$

due to (3). Hence there exist no nonnegative numbers $x, y$, and $z$ such that $f_{1}(x, y, z)=f_{2}(x, y, z)=f_{3}(x, y, z)=0$. Since $P \in(s,-s,-s)$ and it is impossible that $f_{1}(P)=f_{2}(P)=$ $f_{3}(P)=0,(24)$ gives that the sign of $f_{1}(T(P))$ is $s$.

Using Theorem 2, we have the following:

$$
\begin{aligned}
& \text { If } P \in \mathrm{I}(+,-,-) \text {, } \\
& \text { then } T(P) \notin \operatorname{III} \cup \operatorname{IV} \cup \mathrm{V}(-, *, *) .
\end{aligned}
$$

If $P \in \mathrm{V}(-,+,+), \quad$ then $T(P) \notin \mathrm{I}(+,-,-)$.

Remark 3. Suppose that $\Delta t$ satisfies (9) and (15). Let $s \in$ $\{+,-\}$. Then the definitions of $f_{2}$ and $f_{3}$ yield

$$
\begin{aligned}
f_{2}(T(P))= & \left(1-\Delta t a_{22} P_{2}\right) f_{2}(P) \\
& +\Delta t\left\{a_{21} P_{1} f_{1}(P)-a_{23} P_{3} f_{3}(P)\right\}, \\
f_{3}(T(P))= & \left(1-\Delta t a_{33} P_{3}\right) f_{3}(P) \\
& +\Delta t\left\{a_{31} P_{1} f_{1}(P)+a_{32} P_{2} f_{2}(P)\right\},
\end{aligned}
$$

and hence we can also obtain the property as in Theorem 2 . 
(a) If $P \in(s, s,-s)$, then the sign of $f_{2}(T(P))$ is $s$.

(b) If $P \in(s, s, s)$, then the sign of $f_{3}(T(P))$ is $s$.

Using (a) and (b), we can obtain the following:

$$
\begin{aligned}
& \text { If } P \in \operatorname{IV}(-,-,+), \quad \text { then } T(P) \notin \mathrm{V}(-,+,+) \text {, } \\
& \text { If } P \in \operatorname{III}(-,-,-),
\end{aligned}
$$$$
\text { then } T(P) \notin \mathrm{II} \cup \operatorname{IV} \cup \mathrm{V}(*, *,+) \text {. }
$$

It follows from (28) and (29) that every point $P$ in a region cannot move by the map $T$ to regions with three different signs. In the case of regions with two different signs, it is also impossible by the following theorem.

Theorem 4. Suppose that $\Delta t$ satisfies (9), (15), and (16).
(a) If $P \in I I(+,-,+)$, then $T(P) \notin V(-,+,+)$.
(b) If $P \in V(-,+,+)$, then $T(P) \notin I I(+,-,+)$.
(c) If $P \in I I(+,-,+)$, then $T(P) \notin I I I(-,-,-)$.
(d) If $P \in I I I(-,-,-)$, then $T(P) \notin I I(+,-,+)$.
(e) If $P \in I I I(-,-,-)$, then $T(P) \notin V(-,+,+)$.
(f) If $P \in V(-,+,+)$, then $T(P) \notin \operatorname{III}(-,-,-)$.

Proof. (a) Suppose that $T(P) \in \mathrm{V}(-,+,+)$. Then (24) and (30) with $P \in \operatorname{II}(+,-,+)$ give

$$
\begin{aligned}
& \left(1-\Delta t a_{11} P_{1}\right) f_{1}(P)+\Delta t a_{12} P_{2}\left|f_{2}(P)\right| \\
& <\Delta t a_{13} P_{3} f_{3}(P), \\
& a_{23} P_{3} f_{3}(P) \leq a_{21} P_{1} f_{1}(P),
\end{aligned}
$$

respectively. Combining (34) and (35), we obtain

$$
\left(1-\Delta t a_{11} P_{1}\right) f_{1}(P)<\Delta t a_{13} \frac{a_{21} P_{1}}{a_{23}} f_{1}(P) .
$$

Since $f_{1}(P) \geq 0$, inequality (36) is a contradiction to (15). Therefore the proof of $(a)$ is completed.

(b) Suppose that $T(P) \in \mathrm{II}(+,-,+)$. Then the inclusion of $P \in \mathrm{V}(-,+,+)$ gives a contradiction:

$$
\begin{aligned}
0 \leq & f_{1}(T(P)) \\
= & \left(1-\Delta t a_{11} P_{1}\right) f_{1}(P) \\
& -\Delta t\left\{a_{12} P_{2} f_{2}(P)+a_{13} P_{3} f_{3}(P)\right\}<0 .
\end{aligned}
$$

(c) Suppose that $T(P) \in \operatorname{III}(-,-,-)$. Then (24) and (31) with $P \in \mathrm{II}(+,-,+)$ give

$$
\begin{aligned}
& \left(1-\Delta t a_{11} P_{1}\right) f_{1}(P)+\Delta t a_{12} P_{2}\left|f_{2}(P)\right| \\
& \quad \leq \Delta t a_{13} P_{3} f_{3}(P), \\
& \left(1-\Delta t a_{33} P_{3}\right) f_{3}(P) \leq \Delta t a_{32} P_{2}\left|f_{2}(P)\right|,
\end{aligned}
$$

which yield

$$
\left(1-\Delta t a_{33} P_{3}\right) f_{3}(P) \leq \Delta t a_{32} \frac{a_{13} P_{3}}{a_{12}} f_{3}(P) .
$$

Hence, if $f_{3}(P)>0$ or $f_{3}(P)=0$, then

$$
\begin{aligned}
\left(1-\Delta t a_{33} P_{3}\right) & <\Delta t a_{32} \frac{a_{13} P_{3}}{a_{12}} \\
\quad \text { or } f_{i}(P) & =0 \quad(1 \leq i \leq 3),
\end{aligned}
$$

respectively; these are contradictions due to (15) and the fact that there is no solution of the system of equations $f_{i}(x, y, z)=0(i=1,2,3)$.

(d) and (e) are proved by (33).

(f) Suppose that $T(P) \in \operatorname{III}(-,-,-)$. Then (31) with $P \in$ $\mathrm{V}(-,+,+)$ gives

$$
0<f_{3}(P) \leq \frac{\Delta t}{1-\Delta t a_{33} P_{3}} a_{31} P_{1}\left|f_{1}(P)\right| .
$$

It follows from $f_{2}(P)=-r_{2}+a_{21} P_{1}-a_{22} P_{2}-a_{23} P_{3} \geq 0,(5)$, and (3) that

$$
\begin{aligned}
f_{3}(P) \geq & -r_{3}+a_{31} \frac{1}{a_{21}}\left(r_{2}+a_{22} P_{2}+a_{23} P_{3}\right)+a_{32} P_{2} \\
& -a_{33} P_{3} \\
> & \frac{1}{a_{21}}\left\{-a_{21} r_{3}+a_{31} r_{2}+\left(a_{31} a_{23}-a_{21} a_{33}\right) P_{3}\right\} \\
> & \frac{1}{a_{21}}\left(-a_{21} r_{3}+a_{31} r_{2}\right) .
\end{aligned}
$$

Since $-a_{21} r_{3}+a_{31} r_{2}>0$ by (3), inequality (41) with (42) is a contradiction to (16), which completes the proof.

Theorem 5. Suppose that $\Delta t$ satisfies (9). If $P \in V$, then $T^{m_{1}}(P) \notin V$ for some $m_{1}$.

Proof. Suppose that $T^{m}(P) \in \mathrm{V}(-,+,+)$ for all $m$. Then there exist constants $x_{*}, y_{*}$, and $z_{*}$ such that

$$
\begin{aligned}
& x_{*}=\lim _{k \rightarrow \infty} x_{k}^{1} \geq 0, \\
& y_{*}=\lim _{k \rightarrow \infty} x_{k}^{2}>0, \\
& z_{*}=\lim _{k \rightarrow \infty} x_{k}^{3}>0,
\end{aligned}
$$

and so

$$
f_{2}\left(x_{*}, y_{*}, z_{*}\right)=f_{3}\left(x_{*}, y_{*}, z_{*}\right)=0 \text {. }
$$

This is a contradiction since the system of (44) gives that

$$
\begin{aligned}
0 & <\left(a_{22} a_{31}+a_{32} a_{21}\right) y_{*} \\
& =-\left(a_{31} r_{2}-a_{21} r_{3}\right)-\left(a_{23} a_{31}-a_{33} a_{21}\right) z_{*}<0,
\end{aligned}
$$

due to both (3) and (4) with $z_{*}>0$.

The results we obtained are summarized in Table 1.

Finally, using Table 1, we can obtain the following theorem. 
TABLE 1: The regions containing $T(P)$. The symbols I to $\mathrm{V}$ denote the regions defined in (11). The region I in the second column denotes $P \in \mathrm{I}$, the symbol $\times$ at $(P, T(P))=(\mathrm{I}, \mathrm{III})$ means $T(P) \notin \mathrm{III}$, and then the two circles $\circ$ together at both (I, I) and (I, II) denote that $T(P) \in \mathrm{I} \cup \mathrm{II}$ by (28). The symbol $\boldsymbol{X}$ denotes that $P \in \mathrm{V}$ and $T^{m_{1}}(P) \notin$ $\mathrm{V}$ for some $m_{1}$ by Theorem 5 .

\begin{tabular}{|c|c|c|c|c|c|c|c|}
\hline & \multicolumn{5}{|c|}{$T(P)$} & \multirow{2}{*}{$\begin{array}{c}\text { Equation and } \\
\text { theorem }\end{array}$} \\
\hline & & I & II & III & IV & V & \\
\hline \multirow{5}{*}{$P$} & I & $\circ$ & $\circ$ & $\times$ & $\times$ & $x$ & Equation (28) \\
\hline & II & $\circ$ & $\circ$ & $\times$ & $\circ$ & $x$ & $\begin{array}{c}\text { Theorem } 4(\mathrm{c}) \\
\text { and (a) }\end{array}$ \\
\hline & III & $\circ$ & $x$ & $\circ$ & $x$ & $x$ & Equation (33) \\
\hline & IV & $\circ$ & $\circ$ & $\circ$ & $\circ$ & $x$ & Equation (32) \\
\hline & $\mathrm{V}$ & $x$ & $x$ & $\times$ & $\circ$ & $x$ & $\begin{array}{c}\text { Equation (29); } \\
\text { Theorem 4(b) } \\
\text { and (f); and } \\
\text { Theorem } 5\end{array}$ \\
\hline
\end{tabular}

Theorem 6. Suppose that $\Delta t$ satisfies (9), (15), and (16). If $P \in$ $I \cup I I \cup I I I \cup I V \cup V$, then for all sufficiently large $m$

$$
T^{m}(P) \in I \cup I I \cup I I I \cup I V(*,-, *) .
$$

Remark 7. Table 1 and Theorem 6 are obtained for the case that only two planes $f_{i}(x, y, z)=0(i=1,3)$ intersect in the first octant. We can also apply the approach to the other cases and then have a table and a theorem similar to Table 1 and Theorem 6.

\section{Global Stability}

In this section we show that the fixed point $\vartheta=\left(\vartheta_{1}, 0, \vartheta_{3}\right)$ of the map $T$ satisfying (19) is globally stable. Let $\Delta t$ satisfy that

$$
\begin{aligned}
& \left\{a_{i 1}\left(\chi_{1}+\vartheta_{1}\right)+a_{i 2} \chi_{2}+a_{i 3}\left(\chi_{3}+\vartheta_{3}\right)\right\} \Delta t<\frac{1}{2}, \\
& \qquad t<=1,3, \\
& \Delta t<\frac{\min \left\{a_{31} a_{11}, a_{13} a_{33}\right\}}{2 a_{31}\left(a_{12}+A \vartheta_{1} M\right)+2 a_{13}\left(a_{32}+A \vartheta_{3} M\right)},
\end{aligned}
$$

where $A=3 \sum_{1 \leq j \leq 3}\left(a_{1 j}+a_{3 j}\right)$ and $M=6 \max _{1 \leq i, j \leq 3} a_{i j}$.

Theorem 8. Let $\vartheta$ be the point defined in (19). Suppose that $\Delta t$ satisfies (9), (15), (16), (47), and (48). Assume that the initial point $\left(x_{0}^{1}, x_{0}^{2}, x_{0}^{3}\right)$ of the Euler scheme (7) satisfies

$$
\left(x_{0}^{1}, x_{0}^{2}, x_{0}^{3}\right) \in \prod_{1 \leq i \leq 3}\left(0, x_{i}\right)
$$

Then

$$
\lim _{k \rightarrow \infty}\left(x_{k}^{1}, x_{k}^{2}, x_{k}^{3}\right)=\vartheta
$$

Proof. Theorem 6 gives that for all $k$

$$
\left(x_{k}^{1}, x_{k}^{2}, x_{k}^{3}\right) \in \mathrm{I} \cup \operatorname{II} \cup \operatorname{III} \cup \operatorname{IV}(*,-, *) \text {, }
$$

and then (10) gives that for a nonnegative constant $y_{*}$ :

$$
y_{*}=\lim _{k \rightarrow \infty} x_{k}^{2} .
$$

Now we claim that $y_{*}=0$ : suppose, on the contrary, that

$$
y_{*}>0 \text {. }
$$

Applying both (52) and (53) to (7), we have

$$
\lim _{k \rightarrow \infty} f_{2}\left(x_{k}^{1}, x_{k}^{2}, x_{k}^{3}\right)=0,
$$

which implies that there exists a constant $\epsilon_{0}$ such that for all sufficiently large $k$

$$
\begin{aligned}
0 & <\epsilon_{0} \\
& <a_{21} \max \left\{\frac{r_{2}}{a_{21}}-\frac{r_{1}}{a_{11}}, \frac{r_{2}}{a_{21}}-\frac{r_{3}}{a_{31}}\right\}, \\
f_{2}\left(x_{k}^{1}, x_{k}^{2}, x_{k}^{3}\right) & >-\epsilon_{0} .
\end{aligned}
$$

Then (56) and (55) give $x_{k}^{1}>\left(1 / a_{21}\right)\left(r_{2}-\epsilon_{0}\right)>r_{1} / a_{11}$, and so (14) gives

$$
\left(x_{k}^{1}, x_{k}^{2}, x_{k}^{3}\right) \notin \mathrm{I}(+,-,-) .
$$

Using (56), we have

$$
x_{k}^{1}>\frac{1}{a_{21}}\left(r_{2}-\epsilon_{0}+a_{22} x_{k}^{2}+a_{23} x_{k}^{3}\right),
$$

and so it follows from (42) and (55) that for all sufficiently large $k$

$$
f_{3}\left(x_{k}^{1}, x_{k}^{2}, x_{k}^{3}\right)>\frac{1}{a_{21}}\left\{a_{31}\left(r_{2}-\epsilon_{0}\right)-a_{21} r_{3}\right\}>0,
$$

which gives

$$
\left(x_{k}^{1}, x_{k}^{2}, x_{k}^{3}\right) \notin \operatorname{III}(-,-,-) .
$$

Hence (51) with (57) and (60) gives that for all sufficiently large $k$

$$
\left(x_{k}^{1}, x_{k}^{2}, x_{k}^{3}\right) \in \operatorname{II} \cup \operatorname{IV}(*,-,+) .
$$

Both (61) and (53) yield that there exist the two limits:

$$
\begin{aligned}
& x_{*}=\lim _{k \rightarrow \infty} x_{k}^{1}, \\
& z_{*}=\lim _{k \rightarrow \infty} x_{k}^{3}>0,
\end{aligned}
$$

and then

$$
f_{2}\left(x_{*}, y_{*}, z_{*}\right)=f_{3}\left(x_{*}, y_{*}, z_{*}\right)=0 \text {. }
$$

This is a contradiction due to (45) and finally we obtain the claim

$$
\lim _{k \rightarrow \infty} x_{k}^{2}=0
$$


Consider the function $V_{k}$ defined by

$$
V_{k}=a_{31}\left(x_{k}^{1}-\vartheta_{1} \ln x_{k}^{1}\right)+a_{13}\left(x_{k}^{3}-\vartheta_{3} \ln x_{k}^{3}\right) .
$$

Letting $\widehat{a}_{i j}=a_{i j} \Delta t$ and

$$
\begin{aligned}
& \vartheta_{1 k}=\vartheta_{1}-x_{k}^{1}, \\
& \vartheta_{3 k}=\vartheta_{3}-x_{k}^{3},
\end{aligned}
$$

scheme (7) and the fixed point (19) yield

$$
\begin{aligned}
& \Delta x_{k}^{1}=x_{k}^{1}\left(\widehat{a}_{11} \vartheta_{1 k}-\widehat{a}_{12} x_{k}^{2}+\widehat{a}_{13} \vartheta_{3 k}\right), \\
& \Delta x_{k}^{3}=x_{k}^{3}\left(-\widehat{a}_{31} \vartheta_{1 k}+\widehat{a}_{32} x_{k}^{2}+\widehat{a}_{33} \vartheta_{3 k}\right) .
\end{aligned}
$$

Then the mean value theorem gives that for some $\alpha$, $\beta$ with $0<\alpha, \beta<1$

$$
\begin{aligned}
& V_{k+1}-V_{k}=a_{31} \Delta x_{k}^{1}\left(1-\vartheta_{1} \frac{\Delta \ln x_{k}^{1}}{\Delta x_{k}^{1}}\right) \\
& +a_{13} \Delta x_{k}^{3}\left(1-\vartheta_{3} \frac{\Delta \ln x_{k}^{3}}{\Delta x_{k}^{3}}\right) \\
& =a_{31}\left(\widehat{a}_{11} \vartheta_{1 k}-\widehat{a}_{12} x_{k}^{2}+\widehat{a}_{13} \vartheta_{3 k}\right) \\
& \cdot\left(x_{k}^{1}-\vartheta_{1} \frac{x_{k}^{1}}{\alpha \Delta x_{k}^{1}+x_{k}^{1}}\right) \\
& +a_{13}\left(-\widehat{a}_{31} \vartheta_{1 k}+\widehat{a}_{32} x_{k}^{2}+\widehat{a}_{33} \vartheta_{3 k}\right) \\
& \cdot\left(x_{k}^{3}-\vartheta_{3} \frac{x_{k}^{3}}{\beta \Delta x_{k}^{3}+x_{k}^{3}}\right) .
\end{aligned}
$$

Note that

$$
\begin{aligned}
\frac{x_{k}^{1}}{\alpha \Delta x_{k}^{1}+x_{k}^{1}}= & 1-\frac{\alpha\left(a_{11} \vartheta_{1 k}-a_{12} x_{k}^{2}+a_{13} \vartheta_{3 k}\right) \Delta t}{\alpha\left(a_{11} \vartheta_{1 k}-a_{12} x_{k}^{2}+a_{13} \vartheta_{3 k}\right) \Delta t+1} \\
\equiv & 1-\left(C_{1} \vartheta_{1 k}+C_{2} x_{k}^{2}+C_{3} \vartheta_{3 k}\right) \Delta t, \\
\frac{x_{k}^{3}}{\beta \Delta x_{k}^{3}+x_{k}^{3}}= & 1 \\
& -\frac{\beta\left(-a_{31} \vartheta_{1 k}+a_{32} x_{k}^{2}+a_{33} \vartheta_{3 k}\right) \Delta t}{\beta\left(-a_{31} \vartheta_{1 k}+a_{32} x_{k}^{2}+a_{33} \vartheta_{3 k}\right) \Delta t+1} \\
\equiv & 1-\left(C_{4} \vartheta_{1 k}+C_{5} x_{k}^{2}+C_{6} \vartheta_{3 k}\right) \Delta t,
\end{aligned}
$$

where (47) gives

$$
\max _{1 \leq i \leq 6}\left|C_{i}\right|<2 \max _{1 \leq i, j \leq 3} a_{i j}
$$

Now suppose, on the contrary, that $\left(x_{k}^{1}, x_{k}^{2}, x_{k}^{3}\right)$ does not converge to $\left(\vartheta_{1}, 0, \vartheta_{3}\right)$.

Since $\vartheta$ is asymptotically stable by Lemma 1 , the supposition with $\lim _{k \rightarrow \infty} x_{k}^{2}=0$ implies that

$$
\left|\vartheta_{1 k}\right|+\left|\vartheta_{3 k}\right| \text { has a positive lower bound. }
$$

Again, using $\lim _{k \rightarrow \infty} x_{k}^{2}=0$ with (71), we can have that for all sufficiently large $k$

$$
\left|x_{k}^{2}\right|<\Delta t\left(\left|\vartheta_{1 k}\right|+\left|\vartheta_{3 k}\right|\right) .
$$

Then (68) becomes

$$
\begin{aligned}
V_{k+1}-V_{k} \leq & -\Delta t\left(a_{31} a_{11}-C_{7} \Delta t\right) \vartheta_{1 k}^{2} \\
& -\Delta t\left(a_{13} a_{33}-C_{8} \Delta t\right) \vartheta_{3 k}^{2},
\end{aligned}
$$

where

$$
\begin{aligned}
\max \left\{C_{7}, C_{8}\right\}< & 2 a_{31}\left(a_{12}+A \vartheta_{1}\left|C_{123}\right|\right) \\
& +2 a_{13}\left(a_{32}+A \vartheta_{3}\left|C_{456}\right|\right),
\end{aligned}
$$

with $A=3 \sum_{1 \leq j \leq 3}\left(a_{1 j}+a_{3 j}\right)$ and $\left|C_{i j k}\right|=\left|C_{i}\right|+\left|C_{j}\right|+\left|C_{k}\right|$. Hence (73) with (48) becomes

$$
V_{k+1}-V_{k} \leq-C_{9}\left(\vartheta_{1 k}^{2}+\vartheta_{3 k}^{2}\right) \Delta t
$$

for a positive constant $C_{9}$, so that there exists a positive constant $\mathscr{C}$ such that for all sufficiently large $k$

$$
V_{k+1}-V_{\mathrm{k}} \leq-\mathscr{C} \Delta t
$$

and then

$$
V_{k} \leq V_{0}-k \mathscr{C} \Delta t
$$

Hence we have

$$
\lim _{k \rightarrow \infty} V_{k}=\lim _{k \rightarrow \infty}\left(V_{0}-k \mathscr{C} \Delta t\right)=-\infty,
$$

which is a contradiction due to (10).

Remark 9. The global stability in Theorem 8 is obtained for the discrete predator-prey model with one prey and two predators by using both Theorem 6 and the Lyapunov type function $V_{k}$. At first, we show that one predator is extinct by Theorem 6 and then we can apply the function $V_{k}$ which was used in the lower dimensional case: the two-dimensional discrete model with one prey and one predator. Therefore our approach can utilize the methods used in lower dimensional models.

On the other hand, in the case that

$$
\begin{aligned}
& \frac{r_{1}}{a_{11}}<\frac{r_{3}}{a_{31}}<\frac{r_{2}}{a_{21}}, \\
& \frac{r_{1}}{a_{12}}<\frac{r_{3}}{a_{32}}, \\
& \frac{a_{21}}{a_{23}}<\frac{a_{31}}{a_{33}},
\end{aligned}
$$

region II is empty and similarly we can obtain the global stability of the fixed point $\left(r_{1} / a_{11}, 0,0\right)$ of the map $T$ by using Theorems 2-5 without applying a Lyapunov type function like $V_{k}$. 

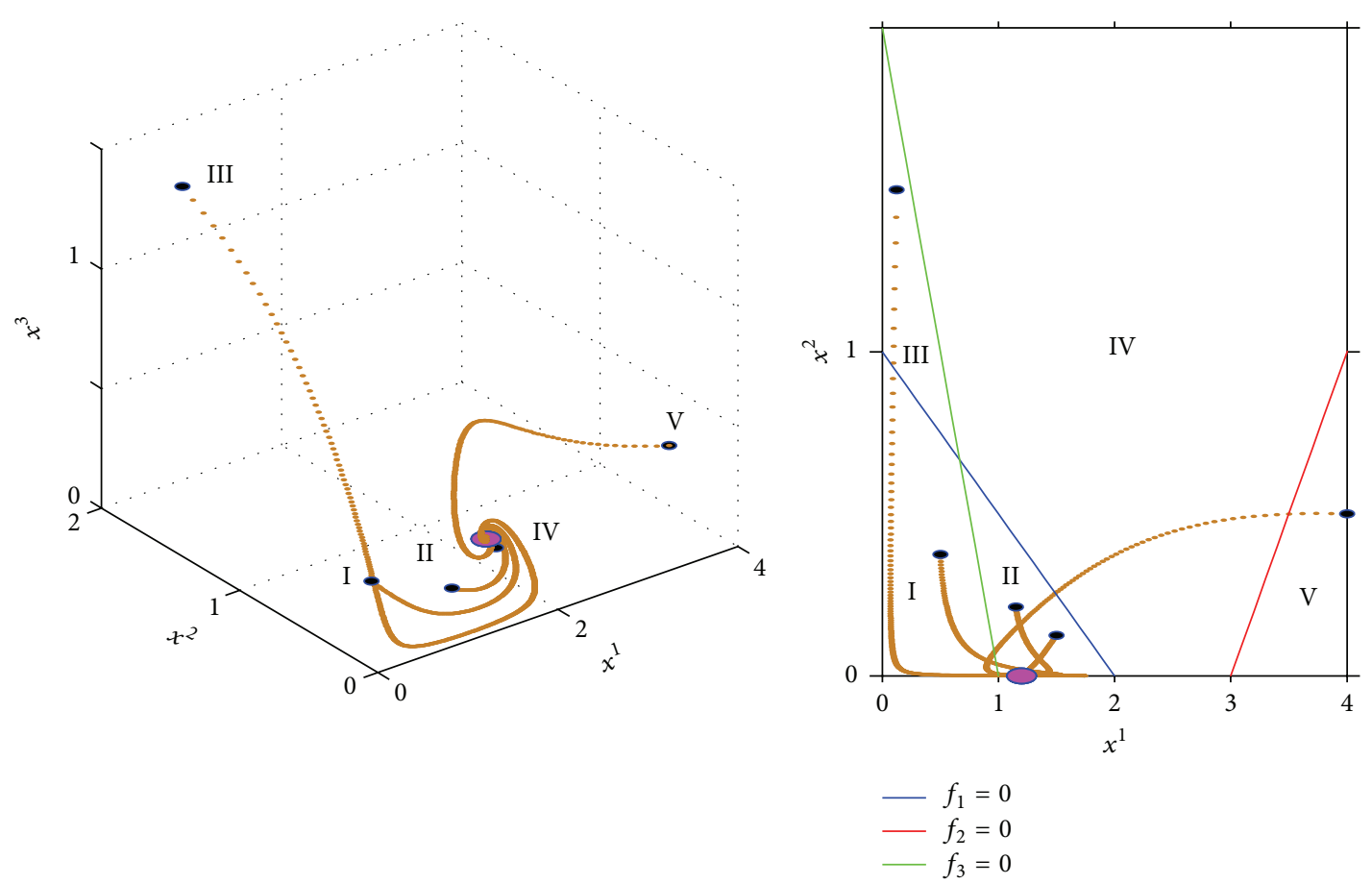

(a)

(b)

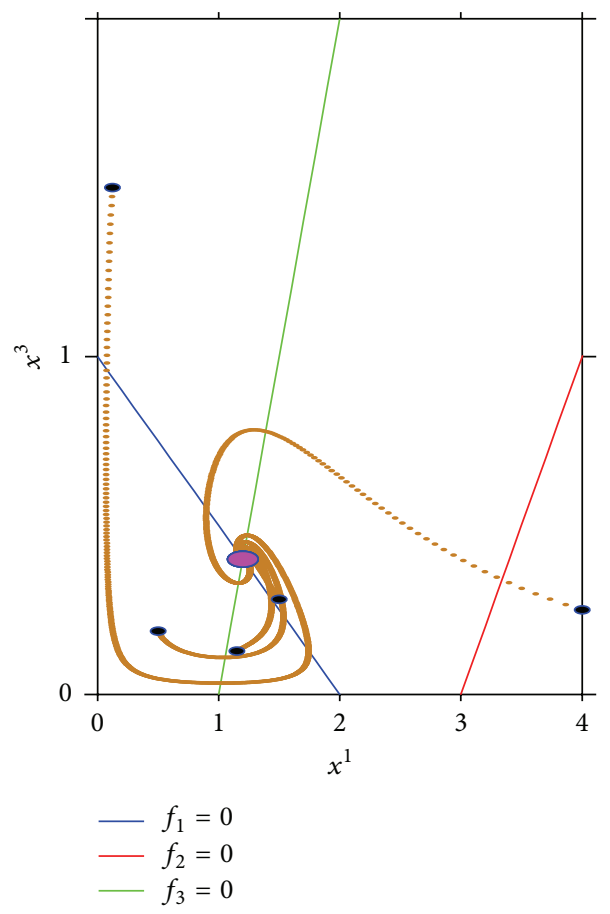

(c)

FIGURE 2: Trajectories for five different initial points denoted by the black circles. The initial points are $(0.5,0.375,0.3562),(1.15,0.2125,0.1281)$, $(0.125,1.5,1.5),(1.5,0.125,0.2813)$, and $(4,0.5,0.25)$, which are contained in regions I, II, III, IV, and V defined in (11), respectively. The brown dotted lines mean the trajectories which converge to $\vartheta=(6 / 5,0,2 / 5)$, denoted by the magenta circle. The three planes $f_{i}=0$ are depicted in Figure 1. 


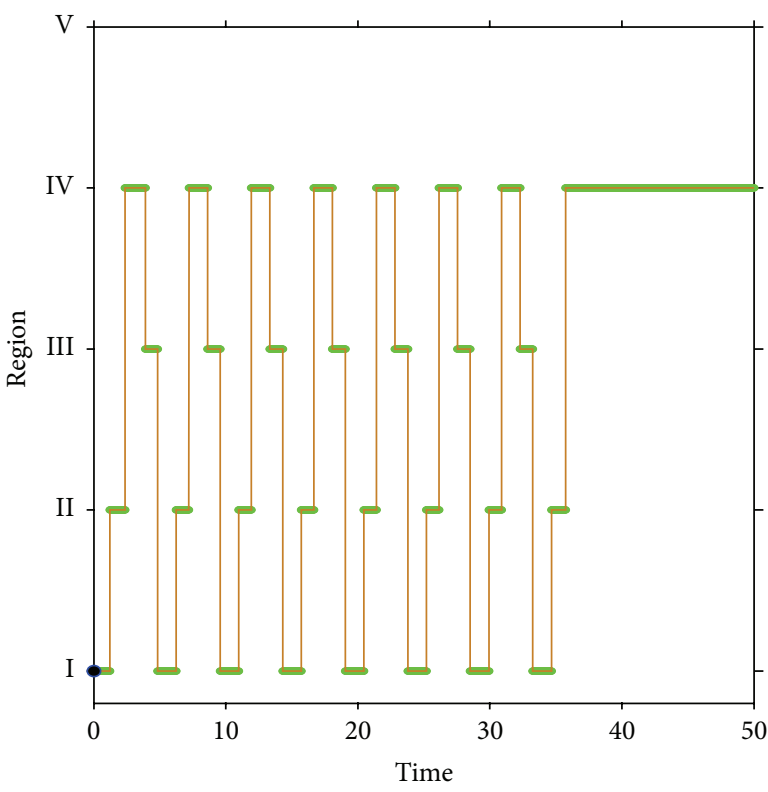

(a)

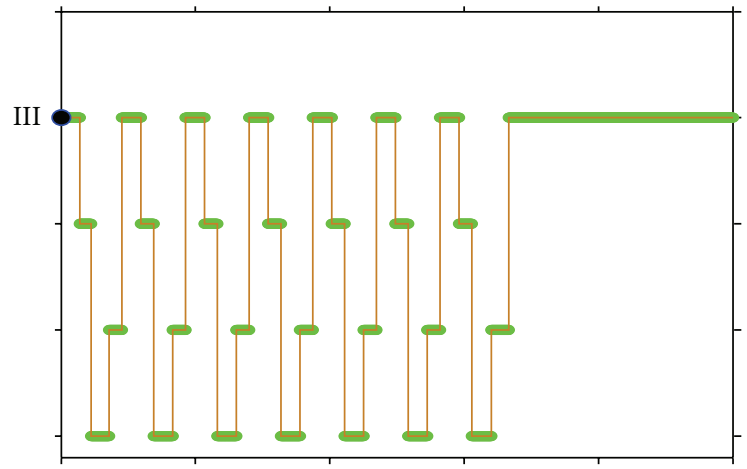

(c)

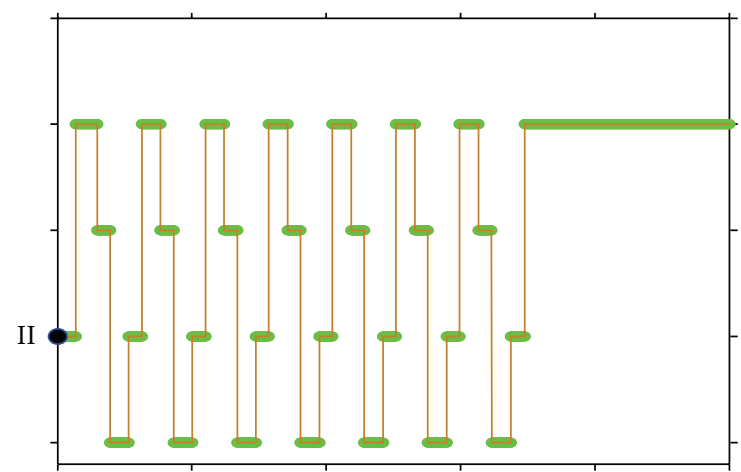

(b)

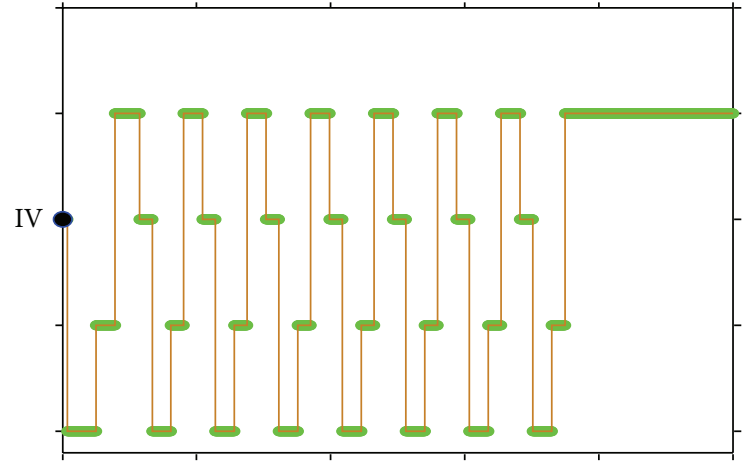

(d)

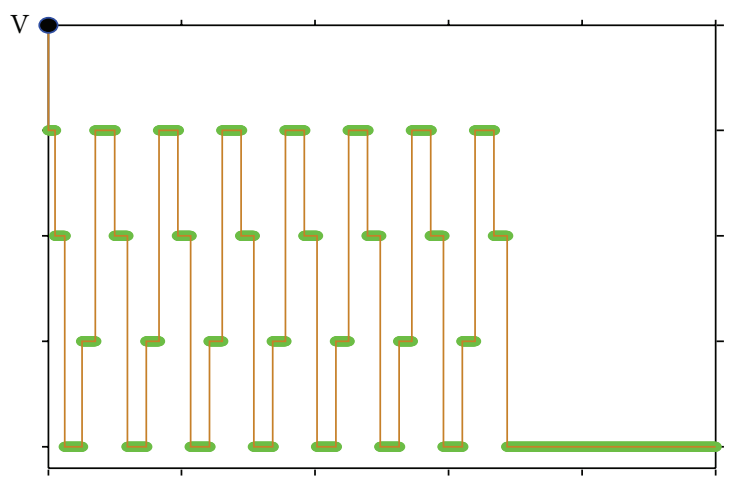

(e)

Figure 3: The region containing $\left(x_{k}^{1}, x_{k}^{2}, x_{k}^{3}\right)$ at time $t=k \Delta t$ for the five trajectories in Figure 2. (a) The initial point $\left(x_{0}^{1}, x_{0}^{2}, x_{0}^{3}\right)$ is $(0.5,0.375$, $0.3562)$, denoted by the black circle and located at $(0, \mathrm{I})$. The green segments denote the regions containing $\left(x_{k}^{1}, x_{k}^{2}, x_{k}^{3}\right)$. The region having $P$ is connected to the region having $T(P)$ by the brown line. The other figures (b), (c), (d), and (e) are similarly obtained by using the different initial points in Figure 2. 


\section{Numerical Examples}

In this section, we consider the Euler difference scheme for (2):

$$
\begin{aligned}
& \Delta x_{k}^{1}=x_{k}^{1}\left(2-x_{k}^{1}-2 x_{k}^{2}-2 x_{k}^{3}\right) \Delta t, \\
& \Delta x_{k}^{2}=x_{k}^{2}\left(-3+x_{k}^{1}-x_{k}^{2}-x_{k}^{3}\right) \Delta t, \\
& \Delta x_{k}^{3}=x_{k}^{3}\left(-2+2 x_{k}^{1}+x_{k}^{2}-x_{k}^{3}\right) \Delta t,
\end{aligned}
$$

where $\Delta t=0.001$ and $k=0,1, \ldots$. Applying Theorem 4.1 in [28] to (80), we can obtain the positivity and boundedness of the solutions. For example,

$$
\left(x_{k}^{1}, x_{k}^{2}, x_{k}^{3}\right) \in(0,4) \times(0,2) \times(0,8) \quad \forall k .
$$

The fixed point $\vartheta$ in (19) becomes $\vartheta=(6 / 5,0,2 / 5)$ which is denoted by the magenta circle in Figures 1 and 2 , and the value $\Delta t=0.001$ satisfies all conditions (9), (15), (16), (47), and (48). Consequently, $\vartheta=(6 / 5,0,2 / 5)$ is globally stable, which is demonstrated in Figure 2 for five different initial points $\left(x_{0}^{1}, x_{0}^{2}, x_{0}^{3}\right)$ contained in regions $\mathrm{I}$ to $\mathrm{V}$, respectively.

In order to verify the results in Table 1, we mark the regions containing $T(P)$ for $P$ located in the five trajectories in Figure 2 and present the result in Figure 3, which demonstrates that the regions containing $T(P)$ follow the rule in Table 1. For example, we can see in Figure 3 that $T(P)$ cannot be contained in $\mathrm{V}$ for every $P \in \mathrm{I} \cup \mathrm{II} \cup \mathrm{III} \cup \mathrm{IV}$, and if $P \in \mathrm{II}$, then $T(P)$ can be contained only in II U IV.

\section{Conclusion}

In this paper, we have developed a new approach to obtain the global stability of the fixed point of a discrete predatorprey system with one prey and two predators. The main idea of our approach is to describe how to trace the trajectories. In this process, we calculate the sign of the rate change of population of species, so that we call our method the sign method. Although we have applied our sign method for the three-dimensional discrete model, the sign method can be utilized for two-dimensional and other higher dimensional discrete models.

\section{Conflict of Interests}

The authors declare that there is no conflict of interests regarding the publication of this paper.

\section{Acknowledgment}

This work was supported by the 2015 Research Fund of University of Ulsan.

\section{References}

[1] J. Alebraheem and Y. Abu-Hasan, "Persistence of predators in a two predators-one prey model with non-periodic solution," Applied Mathematical Sciences, vol. 6, no. 19, pp. 943-956, 2012.
[2] S. B. Hsu and T. W. Huang, "Global stability for a class of predator-prey systems," SIAM Journal on Applied Mathematics, vol. 55, no. 3, pp. 763-783, 1995.

[3] J. Huang, S. Ruan, and J. Song, "Bifurcations in a predatorprey system of Leslie type with generalized Holling type III functional response," Journal of Differential Equations, vol. 257, no. 6, pp. 1721-1752, 2014.

[4] Y. Li and D. Xiao, "Bifurcations of a predator-prey system of Holling and Leslie types," Chaos, Solitons \& Fractals, vol. 34, no. 2, pp. 606-620, 2007.

[5] S. Ruan and D. Xiao, "Global analysis in a predator-prey system with nonmonotonic functional response," SIAM Journal on Applied Mathematics, vol. 61, no. 4, pp. 1445-1472, 2000.

[6] H. K. Baek and D. S. Kim, "Dynamics of a predator-prey system with mixed functional responses," Journal of Applied Mathematics, vol. 2014, Article ID 536019, 10 pages, 2014.

[7] S. Liu and E. Beretta, "A stage-structured predator-prey model of Beddington-DeAngelis type," SIAM Journal on Applied Mathematics, vol. 66, no. 4, pp. 1101-1129, 2006.

[8] S. Shulin and G. Cuihua, "Dynamics of a BeddingtonDeAngelis type predator-prey model with impulsive effect," Journal of Mathematics, vol. 2013, Article ID 826857, 11 pages, 2013.

[9] H. Xiang, X.-Y. Meng, H.-F. Huo, and Q.-Y. Yin, "Stability in a predator-prey model with Crowley-Martin function and stage structure for prey," Applied Mathematics and Computation, vol. 232, pp. 810-819, 2014.

[10] X. Y. Zhou and J. G. Cui, "Global stability of the viral dynamics with Crowley-Martin functional response," Bulletin of the Korean Mathematical Society, vol. 48, no. 3, pp. 555-574, 2011.

[11] P. H. Crowley and E. K. Martin, "Functional responses and interference within and between year classes of a dragony population," Journal of the North American Benthological Society, vol. 8, no. 3, pp. 211-221, 1989.

[12] X. Wang and H. Ma, "A Lyapunov function and global stability for a class of predator-prey models," Discrete Dynamics in Nature and Society, vol. 2012, Article ID 218785, 8 pages, 2012.

[13] H. B. Xiao, "Global analysis of Ivlev's type predator-prey dynamic systems," Applied Mathematics and Mechanics, vol. 28, no. 4, pp. 419-427, 2007.

[14] J. Sugie, "Two-parameter bifurcation in a predator-prey system of Ivlev type," Journal of Mathematical Analysis and Applications, vol. 217, no. 2, pp. 349-371, 1998.

[15] C. Çelik and O. Duman, "Allee effect in a discrete-time predatorprey system," Chaos, Solitons \& Fractals, vol. 40, no. 4, pp. 19561962, 2009.

[16] S. R. J. Jang, "Allee effects in a discrete-time host-parasitoid model," Journal of Difference Equations and Applications, vol. 12, no. 2, pp. 165-181, 2006.

[17] P. C. Tabares, J. D. Ferreira, and V. Sree Hari Rao, "Weak Allee effect in a predator-prey system involving distributed delays," Computational \& Applied Mathematics, vol. 30, no. 3, pp. 675699, 2011.

[18] R. Cui, J. Shi, and B. Wu, "Strong Allee effect in a diffusive predator-prey system with a protection zone," Journal of Differential Equations, vol. 256, no. 1, pp. 108-129, 2014.

[19] J. D. Flores and E. González-Olivares, "Dynamics of a predatorprey model with Allee effect on prey and ratio-dependent functional response," Ecological Complexity, vol. 18, pp. 59-66, 2014. 
[20] N. Shigesada, K. Kawasaki, and E. Teramoto, "Spatial segregation of interacting species," Journal of Theoretical Biology, vol. 79, no. 1, pp. 83-99, 1979.

[21] G. Zhang and X. Wang, "Effect of diffusion and cross-diffusion in a predator-prey model with a transmissible disease in the predator species," Abstract and Applied Analysis, vol. 2014, Article ID 167856, 12 pages, 2014.

[22] Z. Xie, "Cross-diffusion induced Turing instability for a three species food chain model," Journal of Mathematical Analysis and Applications, vol. 388, no. 1, pp. 539-547, 2012.

[23] L. Yang and S. Zhong, "Global stability of a stage-structured predator-prey model with stochastic perturbation," Discrete Dynamics in Nature and Society, vol. 2014, Article ID 512817, 8 pages, 2014.

[24] T. C. Gard, "Persistence in stochastic food web models," Bulletin of Mathematical Biology, vol. 46, no. 3, pp. 357-370, 1984.

[25] M. Vasilova, "Asymptotic behavior of a stochastic Gilpin-Ayala predator-prey system with time-dependent delay," Mathematical and Computer Modelling, vol. 57, no. 3-4, pp. 764-781, 2013.

[26] Q. Din, "Dynamics of a discrete Lotka-Volterra model," Advances in Difference Equations, vol. 2013, article 95, 13 pages, 2013.

[27] T. Wu, "Dynamic behaviors of a discrete two species predatorprey system incorporating harvesting," Discrete Dynamics in Nature and Society, vol. 2012, Article ID 429076, 12 pages, 2012.

[28] S. M. Choo, "Global stability in n-dimensional discrete LotkaVolterra predator-prey models," Advances in Difference Equations, vol. 2014, no. 1, article 11, 17 pages, 2014. 


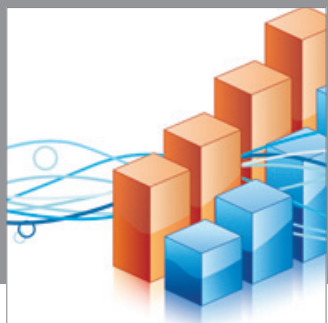

Advances in

Operations Research

mansans

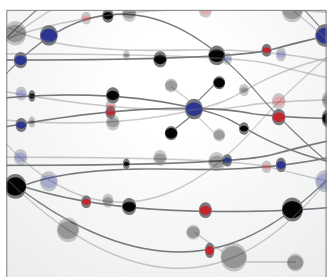

The Scientific World Journal
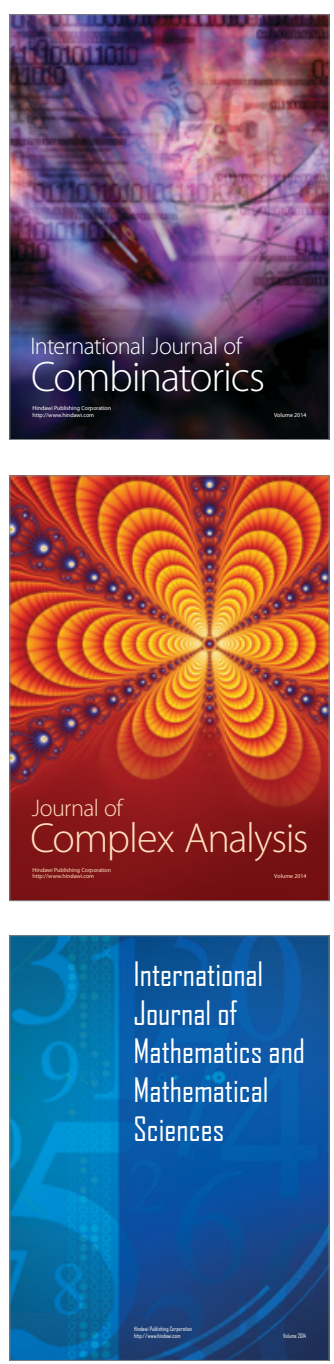
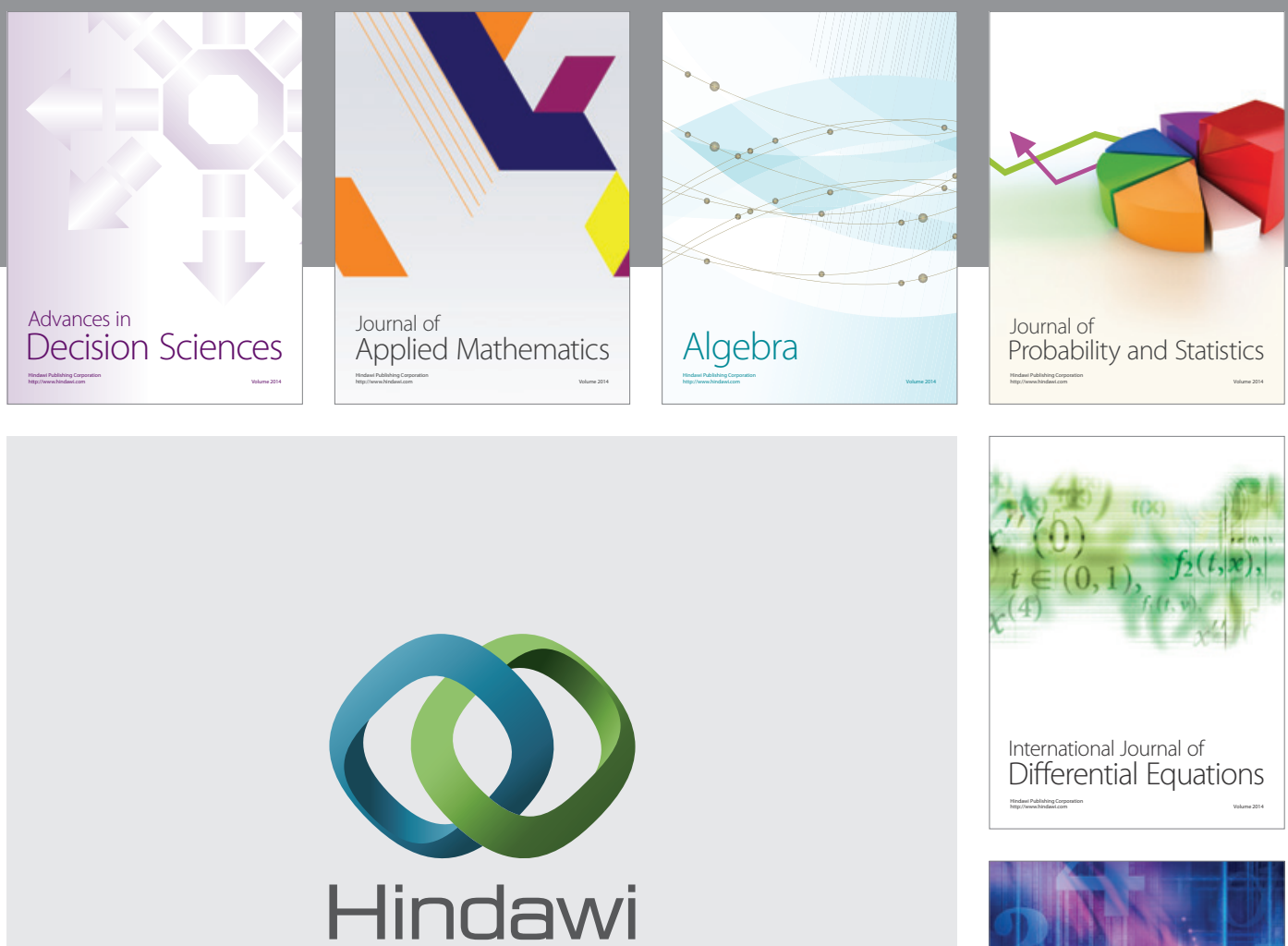

Submit your manuscripts at http://www.hindawi.com
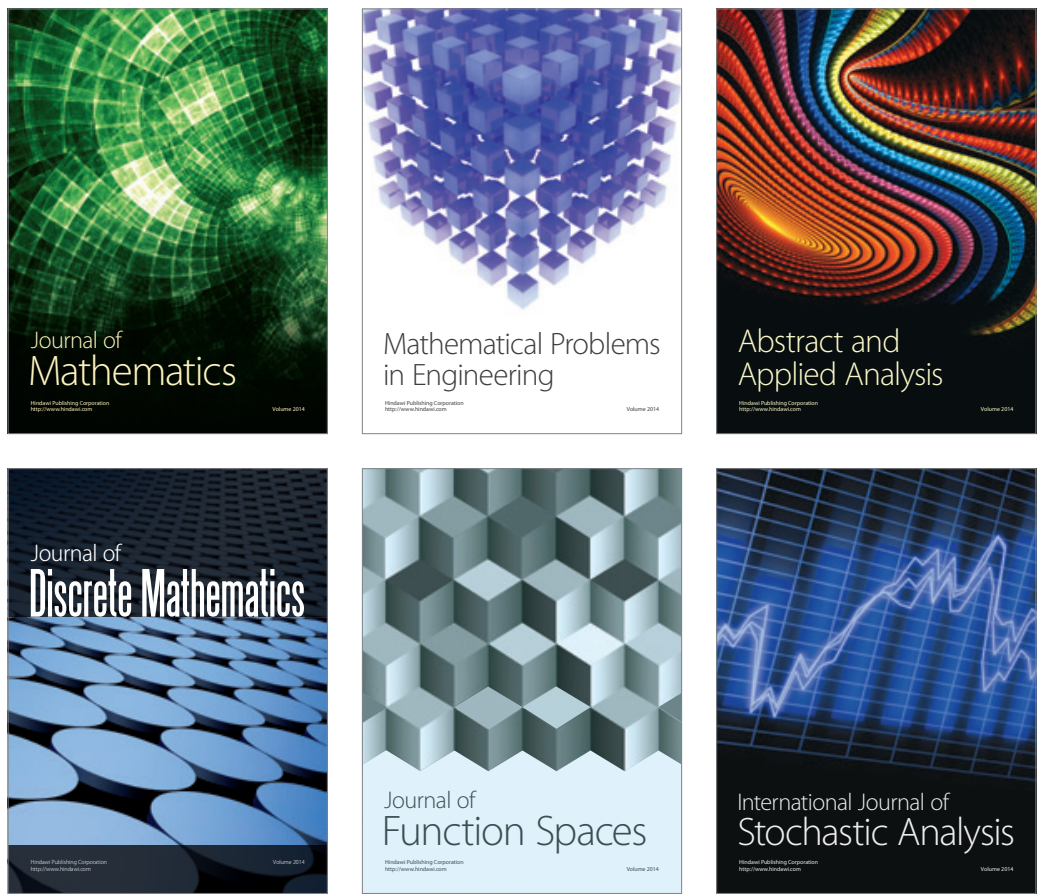

Journal of

Function Spaces

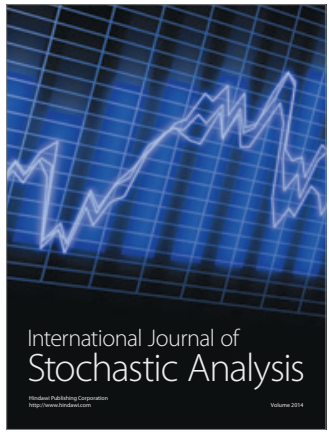

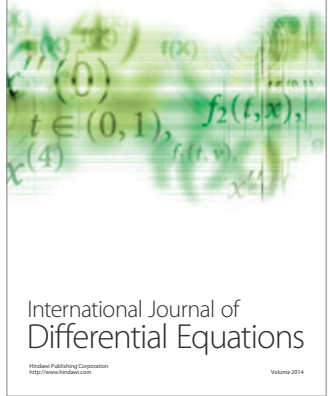
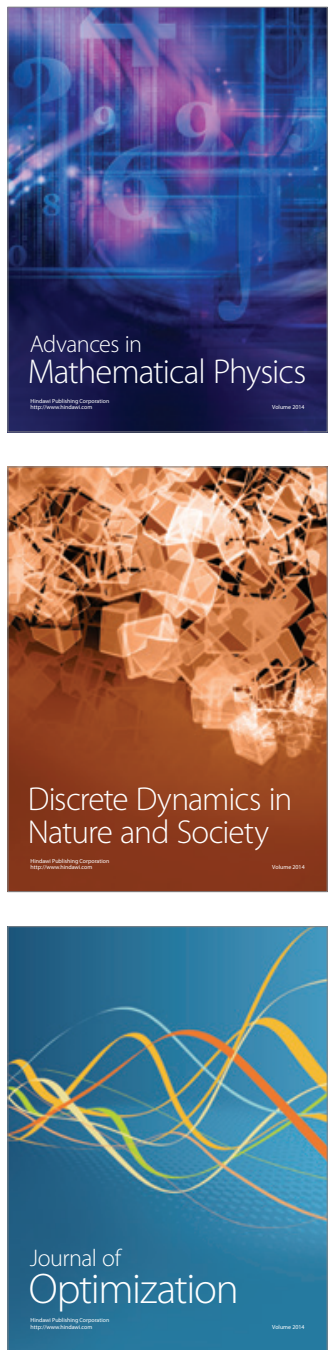